\title{
The impact of COVID-19 on idiopathic scoliosis referrals: cause for concern
}

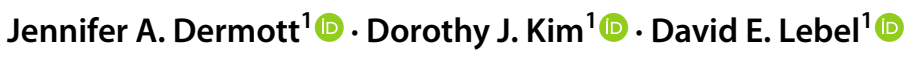

Received: 30 April 2021 / Accepted: 18 September 2021 / Published online: 1 October 2021

(C) Scoliosis Research Society 2021

\begin{abstract}
Study design Retrospective comparative review.

Objective The aim of this study was to determine the short-term impact of the COVID-19 pandemic on the demographic and clinical profile of new idiopathic scoliosis (IS) patients, with a particular focus on treatment and late referral.

Summary of background data The COVID-19 pandemic has limited health-care services and public access and, as a result, the diagnosis and management of non-COVID-19 health conditions have been compromised. Delayed diagnosis of IS may limit conservative treatment options and subsequently, increase a patient's risk of requiring surgical intervention.

Methods The volume of spine referrals received and new IS clinic visits were compared between March 15-October 15, 2019 and the same period in 2020. A chart and radiographic review detailed the patient profile at initial presentation. Descriptive statistics and comparative analyses examined the referral source, curve magnitude, skeletal maturity, and prescribed treatment. Late referrals were those with a curve magnitude $\geq 50^{\circ}$, or $>40^{\circ}$ and Risser 2 or less.

Results During the 2020 study period, the referral volume decreased $76 \%$ and clinic visits $55 \%$. The 2019 cohort was similar in age ( $13.7 \pm 2.1$ years vs $13.3 \pm 2.3$ years, $p=0.08)$, Risser score distribution $(p=0.32)$, menarchal status $(0.07)$, and curve magnitude $\left(37.1^{\circ} \pm 3.8^{\circ}\right.$ vs $\left.39.0^{\circ} \pm 16.0^{\circ}, p=0.22\right)$. During the pandemic, there was an increased proportion of referrals made by pediatricians ( 41 to $54 \%, p=0.01$ ). The proportion of brace prescriptions increased from 30 to $42 \%(p=0.01)$. The proportion of surgical bookings and late referrals were increased but did not reach significance.

Conclusion Despite no significant increase in the magnitude of the curve at initial presentation or the proportion of late referrals, there was a marked decrease in referral volume, over the first 7 months of the COVID-19 pandemic. This suggests that the majority of expected new IS patients remain undiagnosed. A future increase in late referrals should be anticipated. Level of evidence III.
\end{abstract}

Keywords Scoliosis $\cdot$ Pediatric $\cdot$ COVID-19 $\cdot$ Treatment $\cdot$ Late referral

\section{Introduction}

The novel coronavirus disease 2019 (COVID-19) pandemic has impacted every facet of society. The first confirmed case of COVID-19 in North America was January 21, 2020 in Washington State, USA, and 4 days later in Canada. On March 17th, 2020, the government of Ontario declared a state of emergency. Access to health-care services was initially limited to urgent care only. At the Hospital for Sick

Jennifer A. Dermott

jennifer.dermott@sickkids.ca

1 Division of Orthopaedic Surgery, Hospital for Sick Children, 555 University Avenue, Room S229, Toronto, ON M5G 1X8, Canada
Children in Toronto, Canada's largest pediatric academic health sciences center, the gradual restart of scheduled clinical care, up to $65 \%$ capacity, began in June. Clinical capacity increased to $75 \%$ in July, where it remained until the end of this study period.

The limitation to health-care service has been further compounded by families prioritizing their health concerns below their fear of COVID exposure [1]. As a result, diagnosis and management of health conditions, not related to COVID-19, have been compromised. For example, in the first 10 weeks of the pandemic the endoscopy rate in the UK was decreased, on average to $12 \%$, resulting in $58 \%$ of expected cancers not being detected [2]. Delayed diagnosis in progressive conditions may result in increased disease severity at initial presentation. Management options may be 
limited, or more aggressive treatment may need to be considered. Idiopathic scoliosis (IS) is a progressive condition most often diagnosed in adolescence. Delayed IS diagnosis may limit conservative treatment options and, subsequently, increase the risk of requiring surgical intervention.

Scoliosis is defined as a lateral curvature of the spine greater than $10^{\circ}$ (measured with the Cobb method) [3]; it is most common in youth at least 10 years of age. Progression is highly correlated with growth and, during peak growth, curvatures may progress, on average, $1^{\circ}$ per month [4]. Brace treatment significantly decreases the likelihood of a patient requiring surgical intervention [5] and, in accordance with the Scoliosis Research Society's guidelines, is considered most effective for curvatures that measure between $25^{\circ}$ and $40^{\circ}$, in youth that still have growth remaining [6]. Curvatures measuring between $40^{\circ}$ and $50^{\circ}$ are borderline for surgical consideration and surgery is recommended for curves measuring greater than $50^{\circ}$ [7].

IS patients who are likely surgical candidates at initial presentation are considered a 'late' referral. A referral is considered timely if there is no treatment indication or if they are a candidate for bracing. Previous research shows that even prior to COVID-19 restrictions, the volume of 'late' referrals was problematic [8-10]. Anthony et al. recently showed that, in our public health-care system, $28 \%$ of first-time patients presented 'late', whereas only $17 \%$ met the SRS guidelines for brace treatment [8]. The average curve magnitude was near the upper range for optimal brace effectiveness. The authors highlight the implications of late referrals, not only to the patient, but also to the health-care system and an institution's resources [8].

Early COVID-19 publications related to scoliosis have focused primarily on deployment of physicians to the front lines [11-13], prioritization of surgical waitlists based on a temporary hold on elective surgeries [11,12,14-17], use of telemedicine [18], and continued opportunities for education [19] and training programs [11]. To date, the impact of the pandemic on the diagnosis and management of IS patients has not been considered. The aims of this study are to evaluate the extent to which COVID-19 restrictions have impacted (1) the referral volume of idiopathic scoliosis, (2) the patients' clinical and radiographic profile at initial presentation, (3) treatment and (4) the proportion of idiopathic scoliosis patients that present late. We hypothesize that compared to the same time period one-year prior, the number of referrals received during the first 7 months of the COVID-19 pandemic will be greatly reduced, the average curve magnitude at initial presentation will be larger, and the proportion of late referrals will be greater.

\section{Methods}

We conducted a cross-sectional, retrospective cohort study in accordance with the hospital Research Ethics Boardapproved protocol. Patients were identified through our institution's electronic referral platform. Referrals are initiated by a community physician and must include a radiology report that indicates a scoliotic curvature measuring at least $20^{\circ}$. We do not accept referrals for curvatures less than $20^{\circ}$, these are referred back to the family physician to be monitored, or for back pain in the absence of neurologic symptoms and/or explanatory MRI findings.

Two authors [JD and DK], 1 not involved in the patient's care, reviewed the electronic health-care record of all children, between 4 and 17 years of age who were seen for initial consultation in our Spine Clinic between the comparative time periods of: March 15-October 15, 2019 (pre-COVID-19) or March 15-October 15, 2020 (during COVID-19 restrictions). Patient demographic information was recorded. In addition to etiology, the initial clinic note provided the clinical and radiographic findings. If the radiographic details are not clearly stated, the corresponding $\mathrm{X}$-ray was reviewed. Exclusions included a non-scoliosis spinal diagnosis (e.g., \# vertebrae), a non-IS diagnosis (e.g., neuromuscular or syndromic scoliosis), and patients with a history of previous spinal surgery/bracing, or those seeking a second opinion, as we were interested in treatment options at initial presentation.

For this type of study, formal consent is not required.

\section{Outcomes}

The volume of spine-related referrals received and accepted, and the referral source, was recorded. The primary patient outcomes pertained to clinical and radiographic presentation. Demographic information included sex and age at initial presentation. The Cobb angle was recorded for an individual's largest curve. The Risser score [20] was noted and menarchal status, if applicable, was categorized as premenarchal, $<2$, and $\geq 2$ years). Treatment indications were based on curve magnitude in relation to perceived growth potential. A referral was considered "late" if, at initial presentation the patient was likely to undergo surgical intervention at some point in the future, specifically, if the curve magnitude was $50^{\circ}$ or greater, or over $40^{\circ}$ and Risser 2 or less.

\section{Statistical analyses}

Descriptive statistics (mean, standard deviation [SD], range) pertaining to patients' clinical and radiographic 
profile were calculated separately for the 2019 and the 2020 cohort. Frequencies were calculated for categorical data. Welch's $t$ tests were performed on continuous data and chi-squared tests on categorical data.

Statistical significance was accepted at a $p$ value less than 0.05 . Data were analyzed using the web-based stats program R (http://www.R-project.org).

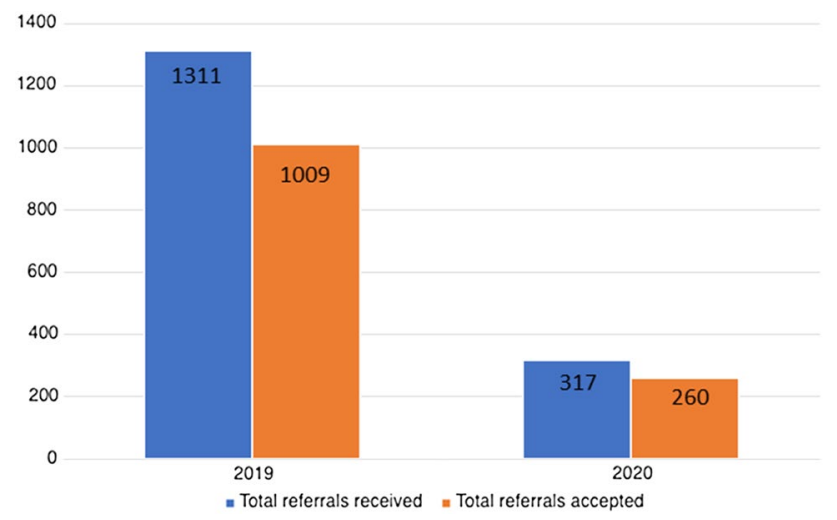

Fig. 1 Idiopathic scoliosis referral volumes (received and accepted) for pre-pandemic (March 16-October 16, 2019) compared to the first 7 months of the pandemic (March 16-October 16, 2020)

\section{Results}

Compared to 2019, the referral volume decreased in 2020 by $76 \%$ ( $n=1311$ vs 317$)$, and the volume of accepted referrals by $74 \%$ ( $n=1009$ vs 260 ) (Fig. 1 ). The proportion of referrals accepted was stable ( 77 vs $82 \%, p=0.5$ ).

In total, 377 patients were eligible for inclusion in this study; 229 patients that were seen for initial consultation in 2019 and 148 in 2020 (-55\%). Table 1 shows that the two groups were similar in age $(13.7 \pm 2.1$ years vs $13.3 \pm 2.3$ years, $p=0.08)$, curve magnitude $\left(37.1^{\circ} \pm 3.8^{\circ}\right.$ vs $\left.39.0^{\circ} \pm 16.0^{\circ}, p=0.22\right)$, Risser score distribution $(p=0.32)$, and menarchal status $(p=0.07)$. Figure 2 illustrates the distribution of curve severity by year. The primary referral source changed in 2020 with an increased proportion of referrals from pediatricians (41-54\%) and a decreased proportion from general practitioners $(47-34 \%$, $p=0.01$ ).

The treatment prescribed at initial visit differed $(p=0.02)$. Figure 3 illustrates that in 2020 a greater proportion of patients were braced (42\% compared to $30 \%$ in 2019) and booked for surgery (15\% compared to $10 \%$ in 2019), and a smaller proportion were monitored (44\% compared to $60 \%$ in 2019). The volume of late referrals increased in 2020 from 25 to $31 \%$ but failed to reach statistical significance $(p=0.1)$.
Table 1 Patient characteristics at initial visit, 2019 versus 2020

\begin{tabular}{llll}
\hline & $2019(n=229)$ & $2020(n=148)$ & $p$ \\
\hline Female, $n(\%)$ & $191(83)$ & $119(80)$ & 0.54 \\
Age, mean $\pm \mathrm{SD}$, years & $13.7 \pm 2.1$ & $13.3 \pm 2.3$ & 0.08 \\
Cobb angle, mean $\pm \mathrm{SD},^{\circ}$ & $37.1 \pm 3.8$ & $39.0 \pm 16.0$ & 0.12 \\
Risser sign, $n(\%)$ & & & 0.32 \\
0 & $52(23)$ & $50(34)$ & \\
1 & $16(7)$ & $11(7)$ & \\
2 & $16(7)$ & $8(5)$ & \\
3 & $20(9)$ & $50(34)$ & \\
4 & $93(41)$ & $16(11)$ & \\
5 & $29(13)$ & $36(24)$ & \\
Menarchal status, $n(\%)$ & & $57(38)$ & $0.01^{*}$ \\
Pre-menarche & $43(19)$ & $20(13)$ & \\
Post-menarche less than 2 years & $92(40)$ & $50(34)$ & \\
Post-menarche greater than 2 years & $52(23)$ & $82(54)$ & \\
Referral source, $(\%)$ & $13(12)$ & \\
General/family practitioner & $93(41)$ & $24(12)$ & \\
Pediatrician & &
\end{tabular}

SD standard deviation

*Significant 


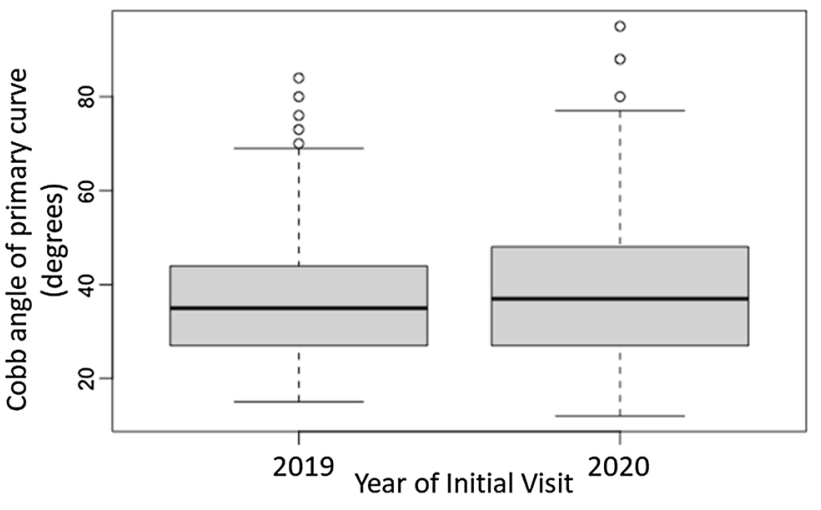

Fig. 2 Boxplot illustrating the distribution of curve magnitude at initial presentation in 2019 compared to 2020. In 2020 there is an increase in the range and interquartile range (IQR) of curve magnitudes at initial presentation. The upper limit (75th percentile) of the curve magnitude IQR is higher and the large magnitude outliers are more extreme

\section{Discussion}

The onset of the COVID-19 pandemic changed the public's utilization of health-care services. It is important that we understand the extent to which these changes have impacted non COVID-19 related disease management in the short-term so that we can prepare for the long-term indirect health consequences of this pandemic. This study focuses on the acute effects on IS referrals and management during the first 7 months of the pandemic. This provides a foundation from which further health-care utilization and patient outcome studies may arise, given that now, COVID-19 variants have emerged and that several countries are experiencing multiple waves of this virus and that its ultimate impact is likely far reaching.

As we hypothesized, our study illustrates a decrease in the volume of IS referrals made to our spine program over the first 7 months of the COVID-19 pandemic. The dramatic $77 \%$ decrease in referral volume implies that the

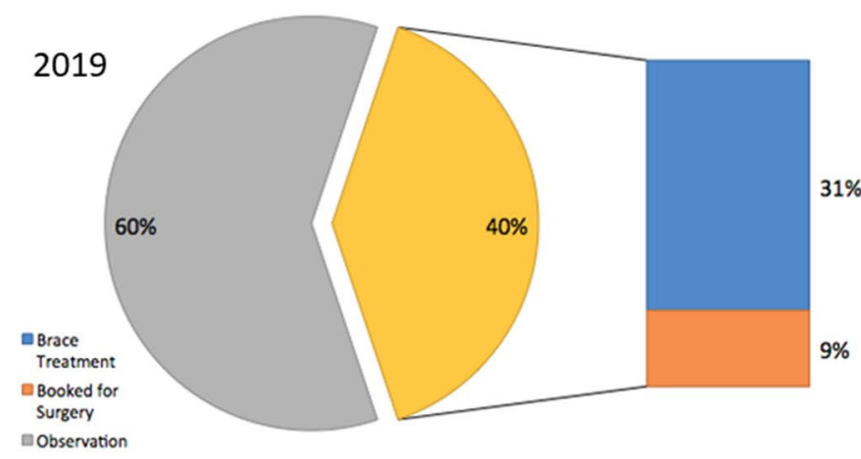

majority of expected IS diagnoses did not occur. Our publicly funded health-care system relies heavily on primary care providers (PCPs) to formally diagnose and refer new patients. Glazier et al. looked at primary care visits in Ontario, between March 11 and July 28, 2020 and found that, compared to 2019 , the largest decrease $(-78.7 \%)$ in office visits and smallest growth in virtual visits, were for individuals $\leq 18$ years [21]. PCPs were defined as family physicians and general practitioners in this study. The decrease in referral volume from this group was similar in our study, at $80 \%$, representing 424 expected IS diagnoses that did not occur. Although IS referrals made by pediatricians decreased by $63 \%$, representing 292 expected IS diagnoses missed, the proportion of IS referrals from pediatricians increased from 41 to $54 \%$. This suggests that, early in the COVID-19 pandemic, the decrease in healthcare utilization was most profound for youth not followed by a pediatrician. Had the IS referral volume from pediatricians decreased in the same manner as it did for family physicians/general practitioners, a further 78 expected IS diagnoses would have been missed in 2020 .

It is possible that this higher proportion of pediatrician referrals factored into the increase in brace eligible patients in 2020, 13 and 12\% increases, respectively. Although the average Risser score and menarchal status did not differ between the study periods, the combination of Risser score and menarchal status, for each individual patient, appears to have increasingly favored brace treatment in 2020 . Nearly $30 \%$ of scoliosis diagnoses are incidental findings by a family physician, GP, or pediatrician [8]. Related to this, it is reasonable that youth who undergo routine well child checkups, which include scoliosis screening by forward bend test, are more likely to have their curvature diagnosed earlier in their adolescent development (i.e., while they are still a brace candidate). Further research is required to explore if the uptake of adolescent well child check-ups differ by the specialty of the primary care provider, and if either of these variables are in fact associated with meeting brace indications.

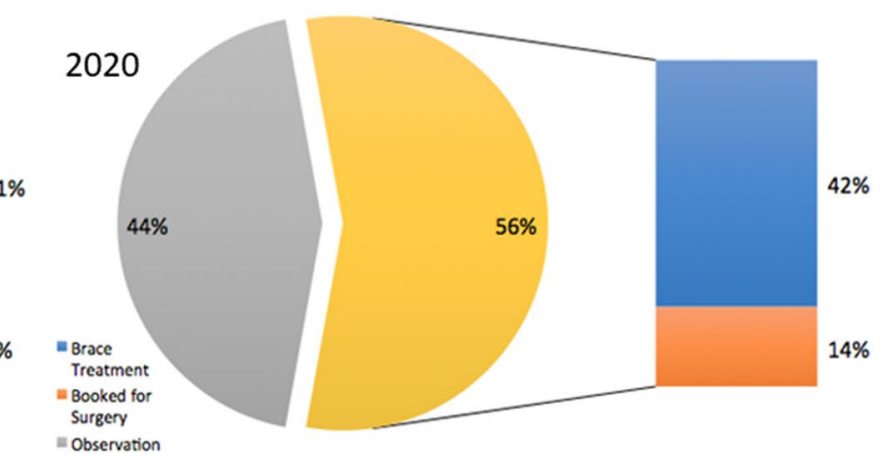

Fig. 3 Distribution of prescribed treatment at the initial clinic consultation, 2019 compared to 2020 
On-line learning and lock down measures, including activity cancellations, have further limited opportunities for early diagnosis. Although scoliosis referrals are ultimately made by PCPs, it is not uncommon for a curvature to first be noted by an individual that is not a health-care professional $[8,22]$. Opportunities for curve recognition include social activities and organized sport. Dance instructors or swim coaches, for example, may notice trunk asymmetry and encourage a family to seek counsel with their PCP. Participation in such activities has been little to none since the onset of the pandemic. This is particularly concerning for adolescents, the most common time for IS diagnosis, as these lifestyle changes are further compounded by their increased desire for privacy at home. Opportunities for curve recognition have been severely limited, both in and out of a medical setting.

At the outset of our study, we hypothesized that our results would show an increase in the average curve magnitude and proportion of late referrals. Despite both outcomes trending in this direction, particularly the proportion of late referrals, which increased from 25 to $31 \%$, the change was not statistically significant. This does not, however, alleviate our grave concern about the long-term impact of this pandemic on the IS population. Unlike some pediatric health events (e.g., trauma related injuries, eating disorders), COVID-19 would not alter the incidence of IS. Considering that approximately three quarters of expected IS diagnoses did not occur over the first 7 months of the pandemic, it is inevitable that the proportion of late referrals will increase, though longer-term follow-up is needed to quantify to what extent. It is unknown how long health-care utilization patterns will remain affected by COVID-19 restrictions but the longer they are, the longer the majority of incident IS cases remain undiagnosed.

Pre-pandemic research illustrates that late referral volumes were an existing issue, often with more children presenting to clinic for the first time as a surgical versus brace candidate [8]. Late referrals inflate surgical waitlists with patients that, had they been diagnosed earlier, may have avoided progression to surgical range by brace treatment [5]. At our institution, waitlists for spine surgery were approximately 18 months prior to COVID-19. The temporary hold on elective surgeries due to the pandemic, then the slow increase in capacity, combined with lower priority given to spinal fusions for IS, will further compound the issue of excessively lengthy waitlists. In the US, there was a predicted backlog of $>1$ million surgical cases due to the deferment of elective surgical cases during the pandemic [23]. Delays to scoliosis surgery greater than 6 months may lead to substantial curve progression and more complex surgical intervention in IS patients who are skeletally immature [24]. Ahn et al. recommended a target of 3 months to surgery to avoid risk of adverse events for skeletally immature patients and those who had larger curvatures [25]. While deformity correction for AIS patients is not considered an essential surgical intervention when triaging orthopedic cases during the COVID-19 pandemic [6, 14], the complications and increased utilization of health-care resources associated with correction of larger curve magnitudes [26] may require reprioritization as we experience now several waves of the pandemic. Future research may evaluate the impact of COVID-19 on spine surgery outcomes, specifically if there was a need to perform more osteotomies, longer fusions, or anterior releases, or, if there were an increase in complication rates, length of post-op hospital stays, or readmissions.

One positive aspect of this pandemic is the significant increase in virtual visits in health care. Telemedicine is not new technology but since the onset of the COVID-19 pandemic, it has been utilized to a much larger extent to limit inperson contact where appropriate. In general, patients have reported high levels of satisfaction with their telemedicine experience during COVID-19 (nearly 88\% 'satisfied, 70\% 'very' satisfied) with $45 \%$ preferring the experience over inperson visits [18]. As we become more familiar with virtual care, we refine our criteria for its use and believe that the use of virtual visits should not hinder PCPs in diagnosing scoliosis. A visual inspection by video camera is often satisfactory to identify clinical features characteristic of scoliosis, such as scapular and/or waistline asymmetry, and rotation in forward bending [27], which is sufficient to determine if an X-ray is indicated. It is not known how much PCPs used virtual care for this purpose during COVID-19 restrictions, but the 77\% decrease in IS referral volume in 2020 suggests that it was underutilized. Given that IS is a diagnosis of exclusion, we continue to favor in-person consultation for initial visits to our spine specialty clinic and have focused our use of virtual care on follow-ups.

Limitations in our study include the inflated volume of IS patients that were seen during the pandemic period compared to the referral rate. Patients that would have been seen in the latter half of March and early April would have been diagnosed and referred to our clinic pre-pandemic. They were included in our study because their initial presentation to our clinic may still have been delayed due to COVID19 , given our clinic volume restrictions and the need to reprioritize clinic visits. This explains why, when referrals decreased by $76 \%$, first-time IS clinic visits only decreased by $55 \%$. Also, the seven-month follow-up time is not long enough to elucidate the full impact of COVID-19 on late referrals and future surgical demands.

\section{Conclusion}

Our results indicate that during the first 7 months of the COVID-19 pandemic, the clinical profile of new IS patients resembled the year prior. However, there was a marked 
reduction in referral volumes. Given the stability of IS incidence, there is concern that the trend towards late referral and increased curve magnitude at initial presentation, will be accentuated in the year(s) to come. This would adversely impact the likelihood of successful brace treatment, increase the volume of patients with surgical indication and may overwhelm surgical programs that are already challenged by lengthy waitlists.

Author contributions Substantial contributions to the conception or design of the work: All. Acquisition of data for work: JD and DK. Analysis of data for the work: JD. Interpretation of data for the work: JD. Drafting the work for important intellectual content: JD and DK. Critical revision of the work for important intellectual content: All. Final approval of the version to be published: All. Agreement to be accountable for all aspects of the work in ensuring that questions related to the accuracy or integrity of any part of the work are appropriately investigated and resolved: All.

Funding None of the authors received financial support for this study.

\section{Declarations}

Conflict of interest All authors declare that they have no conflict of interest.

Ethical approval This retrospective chart review study involving human participants was in accordance with the ethical standards of the 1964 Helsinki Declaration and its later amendments or comparable ethical standards. The Research Ethics Board (REB) of the Hospital for Sick Children approved this study.

Informed consent Informed consent was not required for this type of study.

\section{References}

1. Somekh I, Somech R, Pettoello-Mantovani M et al (2020) Changes in routine pediatric practice in light of Coronavirus 2019 (COVID-19). J Pediatr 224:190-193. https://doi.org/10.1016/j. jpeds.2020.05.053

2. Rutter MD, Brookes M, Lee TJ et al (2021) Impact of the COVID19 pandemic on UK endoscopic activity and cancer detection: a National Endoscopy Database Analysis. Gut 70(3):537-543. https://doi.org/10.1136/gutjnl-2020-322179

3. Kane W (1977) Scoliosis prevalence: a call for a statement of terms. Clin Orthop 126:23-26

4. Lonstein J, Carlson J (1984) The prediction of curve progression in untreated idiopathic scoliosis during growth. J Bone Jt Surg Am 66(7):1062-1071

5. Weinstein SL, Dolan LA, Wright JG et al (2013) Effects of bracing in adolescents with idiopathic scoliosis. N Engl J Med 369(16):1512-1521. https://doi.org/10.1056/NEJMoa1307337

6. Rowe D (2003) Scoliosis research society brace manual. https:// www.srs.org/UserFiles/file/bracing-manual/section1.pdf

7. Lonstein J (2006) Scoliosis: surgical versus non-surgical treatment. Clin Orthop Relat Res 443:248-259

8. Anthony A, Zeller R, Evans C et al (2021) Adolescent idiopathic scoliosis detection and referral trends: impact treatment options. Spine Deform 9(1):75-84. https://doi.org/10.1007/ s43390-020-00182-6

9. Beausejour M, Goulet L, Parent S et al (2013) The effectiveness of scoliosis screening programs: methods for systematic review and expert panel recommendations formulation. Scoliosis 8(1):12. https://doi.org/10.1186/1748-7161-8-12

10. Adobor RD, Riise RB, Sorensen R et al (2012) Scoliosis detection, patient characteristics, referral patterns and treatment in the absence of a screening program in Norway. Scoliosis 7(1):18. https://doi.org/10.1186/1748-7161-7-18

11. Soh TLT, Ding BTK, Yap WMQ et al (2020) Spine surgery and COVID-19: early experiences from Singapore. Spine (Phila Pa 1976) 45(12):786-788. https://doi.org/10.1097/BRS.0000000000 003532

12. Soh TLT, Ho SWL, Yap WMQ et al (2020) Spine surgery and COVID-19: challenges and strategies from the front lines. J Bone Jt Surg Am 102(12):e56. https://doi.org/10.2106/JBJS.20.00503

13. Wang B (2020) The role of spine surgeons in the era of COVID-19 outbreak. Neurospine 17(2):342-343. https://doi.org/10.14245/ns. 2040206.103

14. Anari JB, Baldwin KD, Flynn JM et al (2020) What's important: managing the impact of Coronavirus on pediatric spine surgery. $\mathrm{J}$ Bone Jt Surg Am 102(16):e94. https://doi.org/10.2106/JBJS.20. 00641

15. Jain NS, Wang JC (2020) COVID-19 and the role of spine surgeons. Neurospine 17(2):331-333. https://doi.org/10.14245/ns. 2040202.101

16. DePhillipo N, Larson C, O'Neill O et al (2020) Guidelines for ambulatory surgery centers for the care of surgically necessary/ time-sensitive orthopaedic cases during the COVID-19 pandemic. J Bone Jt Surg Am 102(11):933-936. https://doi.org/10.2106/ JBJS.20.00489

17. Donnally CJ 3rd, Shenoy K, Vaccaro AR et al (2020) Triaging spine surgery in the COVID-19 era. Clin Spine Surg 33(4):129130. https://doi.org/10.1097/BSD.0000000000000988

18. Satin AM, Shenoy K, Sheha ED et al (2020) Spine patient satisfaction with telemedicine during the COVID-19 pandemic: a cross-sectional study. Glob Spine J. https://doi.org/10.1177/21925 68220965521

19. Rasouli JJ, Shin JH, Than KD et al (2020) Virtual spine: a novel, international teleconferencing program developed to increase the accessibility of spine education during the COVID-19 pandemic. World Neurosurg 140:e367-e372. https://doi.org/10.1016/j.wneu. 2020.05.191

20. Risser JC (2010) The classic: the iliac apophysis: an invaluable sign in the management of scoliosis. 1958. Clin Orthop Relat Res 468(3):643-653. https://doi.org/10.1007/s11999-009-1096-Z

21. Glazier RH, Green ME, Wu FC et al (2021) Shifts in office and virtual primary care during the early COVID-19 pandemic in Ontario, Canada. CMAJ 193(6):e200-e210. https://doi.org/10. 1503/cmaj.202303

22. Kenner P, McGrath S, Woodland P (2019) What factors influence delayed referral to spinal surgeon in adolescent idiopathic scoliosis? Spine (Phila Pa 1976) 44(22):1578-1584. https://doi.org/10. 1097/BRS.0000000000003146

23. Jain A, Jain P, Aggarwal S (2020) SARS-CoV-2 impact on elective orthopaedic surgery: implications for post-pandemic recovery. J Bone Jt Surg Am 102(13):e68. https://doi.org/10.2106/JBJS.20. 00602

24. Ramo B, Tran DP, Reddy A et al (2019) Delay to surgery greater than 6 months leads to substantial deformity progression and increased intervention in immature adolescent idiopathic scoliosis (AIS) patients: a retrospective cohort study. Spine Deform 7(3):428-435. https://doi.org/10.1016/j.jspd.2018.09.012

25. Ahn H, Kreder H, Mahomed N et al (2011) Empirically derived maximal acceptable wait time for surgery to treat adolescent 
idiopathic scoliosis. CMAJ 183(9):e565-e570. https://doi.org/ 10.1503/cmaj.101511

26. Miyanji F, Slobogean GP, Samdani AF et al (2012) Is larger scoliosis curve magnitude associated with increased perioperative health-care resource utilization?: a multicenter analysis of
325 adolescent idiopathic scoliosis curves. J Bone Jt Surg Am 94(9):809-813. https://doi.org/10.2106/JBJS.J.01682

27. Satin AM, Lieberman IH (2020) The virtual spine examination: telemedicine in the era of COVID-19 and beyond. Glob Spine J 11(6):966-974. https://doi.org/10.1177/2192568220947744 